\title{
Randomized Play-the-Winner Rule in Two-Period Two-Treatment Repeated Measurement Design
}

\author{
Uttam Bandyopadhyay $^{1}$, Atanu Biswas ${ }^{2}$, and Shirsendu Mukherjee ${ }^{3}$ \\ ${ }^{1}$ University of Calcutta, India \\ ${ }^{2}$ Indian Statistical Institute, Kolkata, India \\ ${ }^{3}$ Behala College, Kolkata, India
}

\begin{abstract}
In the clinical trial randomized play-the-winner rule is used with a goal to allocate more patients to the better treatment in course of sampling. Here we provide an application of this sampling scheme in repeated measurement design. We concentrate on the simplest set up, i.e., on two treatments and two periods. We study, both numerically and theoretically, several exact and limiting properties of this design. We consider some related inferential problems. Finally we use a real data set to illustrate the applicability of our proposed design.
\end{abstract}

Zusammenfassung: In einer klinischen Studie wird die randomisierte playthe-winner Regel mit dem Ziel verwendet, im Zuge der Stichprobenerhebung mehr Patienten der besseren Behandlungsgruppe zuzuweisen. Hier bieten wir eine Anwendung dieses Stichprobenschemas in einem Versuchsplan mit Messwiederholung. Wir konzentrieren uns dabei auf das einfachste Setup, d.h. auf zwei Behandlungen und zwei Perioden. Wir untersuchen sowohl numerisch als auch exakt diverse exakte und asymptotische Eigenschaften dieses Versuchsplans. Weiters betrachten wir einige zugehörige Inferenzprobleme. Schließlich verwenden wir eine realen Datensatz, um die Anwendbarkeit unseres vorgeschlagenen Plans zu illustrieren.

Keywords: Carry-over Effect, Limiting Proportion of Allocation, Maximum Likelihood Estimates, Response-Adaptive Designs, Urn Model.

\section{Introduction}

A response-adaptive treatment allocation design for a clinical trial attempts to place the majority of patients on the treatment that appears more successful, depending on the history of allocations and responses of patients already treated. In case of dichotomous responses, one of the most popular response-adaptive design is Randomized Play-theWinner (RPW) rule introduced by Wei and Durham (1978), which is a modification of Zelen's PW rule (see Zelen, 1969). This rule can be illustrated by an urn model as follows. We start with an urn having $2 \alpha$ balls, $\alpha$ balls of type $A$ and $\alpha$ balls of type $B$. We treat any entering patient by drawing a ball from the urn and replace the ball immediately to the urn. If the patient has a successful response, we add an additional $\beta$ balls of the same type to the urn. On the other hand, if the treatment fails, we add an additional $\beta$ balls of the opposite type to the urn. The idea here is to skew the allocation proportion 
in favor of the treatment that has better performance in course of the trial. Some further works in this direction are, for example, due to Wei $(1979,1988)$, Wei, Smythe, Lin, and Park (1990), Rosenberger (1993, 1996), Bandyopadhyay and Biswas (1996, 1997, 1999, 2001), Ivanova, Rosenberger, Durham, and Flournoy (2000), Hu and Rosenberger (2006), Liang and Carriere (2008). Real life applications of RPW rule are discussed by Bartlett et al. (1985), Reiertsen et al. (1993, 1996); Reiertsen, Larsen, and Solhaug (1998), Tamura, Faries, Andersen, and Heiligenstein (1994), Biswas and Dewanji (2004).

In all the above works and in almost all the works available on the response-adaptive design are concerned with the single period trial. But in case of chronic disease (e.g. asthma, hypertension, rheumatoid arthritis), where one's first evaluation of treatment efficacy is concerned with measuring short-term relief of signs or symptoms, there is a possibility of giving each patient a series of two or more treatments over separate equal periods of time. These repeated measurement designs, better known as crossover or changeover designs, are discussed by many authors, including Grizzle (1965), Brown (1980), Kunert (1991), Senn (1993), Vonesh and Chinchilli (1996, ch.4), Kushner (1997), Carriere and Huang (2000), Jones and Kenward (2003). The main advantage of a crossover trial is that the treatments are compared within-subjects and such within-subject studies allow a more precise comparison of treatments. Real life applications of crossover trial are discussed by, among others, Taka and Armitage (1983), Matthews (1989). In case of continuous response Liang and Carriere (2009) develop a response-adaptive crossover design for clinical trials. But there is hardly any literature related to crossover design using adaptation when the response variable is binary or discrete. An attempt towards this direction on two treatments and on two periods is made by Bandyopadhyay, Biswas, and Mukherjee $(2007,2009)$ for correlated binary responses using a combination of randomized play-the-winner (RPW) and play-the-winner (PW) rules. Such a design, by using an RPW rule, allocates an incoming patient to the first dose of a treatment depending only on the history of the first dose allocations and responses of the treatments. But the allocation of the second dose of a treatment to a patient depends only on his history of allocation and response from the first dose. That is, PW rule is used for each second dose. Thus this rule is a combination of probabilistic and deterministic rules. In the present work we modify Bandyopadhyay et al. $(2007,2009)$ by incorporating all the available data history (corresponding to both first and second doses of the treatments) to get any future first dose allocation. Here we consider a general model for possible carry-over effects where a carry-over effect is defined as the effect of the treatment from the previous time period on the response at the current time period.

In the present work we develop a response-adaptive design for a crossover trial based on randomized play-the-winner principle. The layout of the paper is as follows. Section 2 contains notations and preliminaries. The proposed methodology along with some related exact and asymptotic results are given in Section 3. As a follow up of the rule, we carry out separate tests for treatment effects and carry-over effects. We discuss this in Section 4. Some performance characteristics of the proposed design are discussed in Section 5. Section 6 provides an example of application of our approach. Finally Section 7 concludes. 


\section{Notations and Preliminaries}

We consider two treatments denoted by $A$ and $B$. The number of patients (experimental units) to be examined is $n$, a pre-fixed positive integer. Patients arrive in a clinic sequentially and treated by $A$ or $B$. Each patient is treated twice according to some nature of crossover trial with adaptation. In order to formalize our proposed design, we first introduce the following. For the $i$-th patient, we define the indicator variables $\left\{\delta_{i}, Z_{i}, \tau_{i}, U_{i}\right\}$, where $\delta_{i}\left(\tau_{i}\right)=1$ or 0 as the $i$-th patient receives the first (second) dose of $A$ or $B$, $Z_{i}\left(U_{i}\right)=1$ or 0 as a success or a failure results from the $i$-th patient receiving the first (second) dose of a treatment.

We now consider the following notations and probability models:

- $\bar{\delta}_{i}=1-\delta_{i}, \bar{\tau}_{i}=1-\tau_{i}, \bar{Z}_{i}=1-Z_{i}, \bar{U}_{i}=1-U_{i}$.

- $P\left(Z_{i}=1 \mid \delta_{i}=1\right)=p_{A}, P\left(Z_{i}=1 \mid \delta_{i}=0\right)=p_{B}$.

- $P\left(U_{i}=1 \mid \tau_{i}=1\right)=\phi_{A}, P\left(U_{i}=1 \mid \tau_{i}=0\right)=\phi_{B}$.

- $q_{j}=1-p_{j}, \psi_{j}=1-\phi_{j}, j=A, B$.

- First dose indicators and responses upto the $k$-th patient are represented by

$$
\delta_{(k)}=\left(\delta_{1}, \ldots \delta_{k}\right), \quad Z_{(k)}=\left(Z_{1}, \ldots, Z_{k}\right) .
$$

- Second dose indicators and responses upto the $k$-th patient are similarly represented by $\tau_{(k)}$ and $U_{(k)}$, respectively.

- Sums of the allocations corresponding to the treatments $A$ and $B$ for the first and second dose, upto the $k$-th patient, are defined by $N_{1 A}(k)=\sum_{i=1}^{k} \delta_{i}, N_{1 B}(k)=$ $\sum_{i=1}^{k} \bar{\delta}_{i}, N_{2 A}(k)=\sum_{i=1}^{k} \tau_{i}$, and $N_{2 B}(k)=\sum_{i=1}^{k} \bar{\tau}_{i}$, respectively.

- Sums of responses upto the $k$-th patient for the first dose are defined by $S_{A k}=$ $\sum_{i=1}^{k} \delta_{i} Z_{i}, \bar{S}_{A k}=\sum_{i=1}^{k} \delta_{i} \bar{Z}_{i}, S_{B k}=\sum_{i=1}^{k} \bar{\delta}_{i} Z_{i}, \bar{S}_{B k}=\sum_{i=1}^{k} \bar{\delta}_{i} \bar{Z}_{i}$. Replacing $S$ by $T, \delta_{i}$ by $\tau_{i}$ and $Z_{i}$ by $U_{i}$, we get the corresponding sums for the second dose.

Here we see that the response probabilities in the second time point differ from $p_{A}$ and $p_{B}$, which indicate the presence of some effects other than that of the treatment. The success probabilities of the treatments as measured in one period may differ from those measured in a later period because of a treatment-by-period interaction effect or a carryover effect. In our case of two-period two-treatment crossover design the carry-over and treatment-by-period interaction are aliased with each other and can not be estimated separately. Hence the difference between $p_{A}$ and $\phi_{A}$ and that of $p_{B}$ and $\phi_{B}$ can be attributed to either a carry-over effect or an interaction effect between the treatment and the period.

\section{Proposed Allocation Design}

The proposed rule can be described by an urn model as follows: Consider an urn having balls of two different types marked by $A$ and $B$. We start with $\gamma$ balls of each type. When the first patient enters the system, he is treated twice. At each time we draw a ball at random from the urn and replaced it immediately and the treatment assignment is done according to the type of the ball drawn. That is, as in two-treatment single period RPW 
rule, we assign the first patient to a treatment with probability 0.5 at each period. Based on his allocations and responses at two periods, the urn is updated by using RPW principle as described earlier. Then the second patient enters the study and is treated by drawing a ball at random from the urn with replacements. After getting his response at the first dose of the treatment we update the urn again by RPW principle and he is allotted to a treatment for the second time. Then the 3rd patient enters the study and he is treated twice by the aforesaid procedure and so on. All that we here need is the availability of the responses from the $i$-th patient at both the doses before the entry of the $(i+1)$-st patient. With the notations, as described in Section 2, we get

$$
P\left[\delta_{1}=1\right]=\frac{1}{2}, \quad P\left[\tau_{1}=1\right]=\frac{1}{2},
$$

and for $k \geq 1$,

$$
\begin{gathered}
P\left[\delta_{k+1}=1 \mid \delta_{(k)}, Z_{(k)}, \tau_{(k)}, U_{(k)}\right]=\frac{\gamma+\beta\left(S_{A k}+\bar{S}_{B k}+T_{A k}+\bar{T}_{B k}\right)}{2 \gamma+2 k \beta)}, \\
P\left[\tau_{k+1}=1 \mid \delta_{(k+1)}, Z_{(k+1)}, \tau_{(k)}, U_{(k)}\right]=\frac{\gamma+\beta\left(S_{A k+1}+\bar{S}_{B k+1}+T_{A k}+\bar{T}_{B k}\right)}{2 \gamma+(2 k+1) \beta} .
\end{gathered}
$$

From (1) and (2), after some algebraic manipulations, we successively get $d_{1}, d_{2}, \ldots$ and $t_{1}, t_{2}, \ldots$ as

$$
\begin{aligned}
& P\left(\delta_{k+1}=1\right)=\frac{1}{2}+d_{k+1}, \quad k \geq 0, \\
& P\left(\tau_{k+1}=1\right)=\frac{1}{2}+t_{k+1}, \quad k \geq 0,
\end{aligned}
$$

where $d_{1}=0, t_{1}=0$ and, more generally, $d_{k}$ 's and $t_{k}$ 's are successively related by

$$
d_{k+1}=\frac{\beta}{2 \gamma+2 k \beta}\left\{\frac{k}{2}\left(p_{A}+\phi_{A}-p_{B}-\phi_{B}\right)+\left(p_{A}+p_{B}-1\right) \sum_{i=1}^{k} d_{i}+\left(\phi_{A}+\phi_{B}-1\right) \sum_{i=1}^{k} t_{i}\right\}
$$

and

$$
t_{k+1}=\frac{\beta\left(p_{A}-p_{B}\right)}{2 \gamma+(2 k+1) \beta}+\frac{2 \gamma+\beta\left(2 k-1+p_{A}+p_{B}\right)}{2 \gamma+(2 k+1) \beta} d_{k+1} .
$$

The limiting allocation proportions corresponding to the treatment $A$ in two periods can be obtained by the following result:

Result 3.1 As $n \rightarrow \infty$, almost surely,

$$
\frac{N_{1 A}(n)}{n} \rightarrow \xi, \quad \frac{N_{2 A}(n)}{n} \rightarrow \xi,
$$

where

$$
\xi=\frac{q_{B}+\psi_{B}}{q_{A}+\psi_{A}+q_{B}+\psi_{B}} .
$$

Proof: See Appendix A. 
The following result provides the limiting allocation proportions corresponding to the treatment combinations $A A, A B, B A$ and $B B$ :

Result 3.2 As $n \rightarrow \infty$, almost surely,

$$
\begin{aligned}
& \text { (i) } \frac{1}{n} \sum_{j=1}^{n} \delta_{j} \tau_{j} \rightarrow \xi^{2}, \\
& \text { (ii) } \frac{1}{n} \sum_{j=1}^{n} \delta_{j}\left(1-\tau_{j}\right) \rightarrow \xi(1-\xi), \\
& \text { (iii) } \frac{1}{n} \sum_{j=1}^{n}\left(1-\delta_{j}\right) \tau_{j} \rightarrow \xi(1-\xi), \\
& \text { (iv) } \frac{1}{n} \sum_{j=1}^{n}\left(1-\delta_{j}\right)\left(1-\tau_{j}\right) \rightarrow(1-\xi)^{2} .
\end{aligned}
$$

Proof: See Appendix B.

Under the proposed design, the loglikelihood function, except for the numerical constant, based on $n$ observations is

$$
\begin{aligned}
L_{A}^{\star} & =\sum_{i=1}^{n} \delta_{i} Z_{i} \log p_{A}+\sum_{i=1}^{n} \delta_{i} \bar{Z}_{i} \log q_{A}+\sum_{i=1}^{n} \bar{\delta}_{i} Z_{i} \log p_{B}+\sum_{i=1}^{n} \bar{\delta}_{i} \bar{Z}_{i} \log q_{B} \\
& +\sum_{i=1}^{n} \tau_{i} U_{i} \log \phi_{A}+\sum_{i=1}^{n} \tau_{i} \bar{U}_{i} \log \psi_{A}+\sum_{i=1}^{n} \bar{\tau}_{i} U_{i} \log \phi_{B}+\sum_{i=1}^{n} \bar{\tau}_{i} \bar{U}_{i} \log \psi_{B}
\end{aligned}
$$

Consequently, the maximum likelihood (ML) estimates of $p_{A}, p_{B}, \phi_{A}$ and $\phi_{B}$ are given by

$$
\hat{p}_{A}=\frac{\sum_{i=1}^{n} \delta_{i} Z_{i}}{\sum_{i=1}^{n} \delta_{i}}, \quad \hat{p}_{B}=\frac{\sum_{i=1}^{n} \bar{\delta}_{i} Z_{i}}{\sum_{i=1}^{n} \bar{\delta}_{i}}, \quad \hat{\phi}_{A}=\frac{\sum_{i=1}^{n} \tau_{i} U_{i}}{\sum_{i=1}^{n} \tau_{i}}, \quad \hat{\phi}_{B}=\frac{\sum_{i=1}^{n} \bar{\tau}_{i} U_{i}}{\sum_{i=1}^{n} \bar{\tau}_{i}} .
$$

These estimates are consistent for the respective parameters. The problem of getting a zero divisor can easily be overcome by using the same technique as in Cox and Snell (1989, p.32). See also Geraldes, Melfi, Page, and Zhang (2006) in this connection. Then the following result gives the asymptotic joint distribution of $\hat{p}_{A}, \hat{p}_{B}, \hat{\phi}_{A}$ and $\hat{\phi}_{B}$.

Result 3.3 Let $\mathrm{T}$ be a 4-component vector with elements

$$
\begin{array}{ll}
T_{1}=\sum_{i=1}^{n} \delta_{i}\left(Z_{i}-p_{A}\right), & T_{2}=\sum_{i=1}^{n} \bar{\delta}_{i}\left(Z_{i}-p_{B}\right), \\
T_{3}=\sum_{i=1}^{n} \tau_{i}\left(U_{i}-\phi_{A}\right), & T_{4}=\sum_{i=1}^{n} \bar{\tau}_{i}\left(U_{i}-\phi_{B}\right) .
\end{array}
$$

Then as $n \rightarrow \infty$,

$$
\frac{1}{\sqrt{n}} \mathbf{T} \stackrel{\mathcal{D}}{\longrightarrow} N_{4}(\mathbf{0}, \boldsymbol{\Sigma})
$$


where the elements $\sigma_{i i^{\prime}}$ of $\Sigma$ are given by

$$
\begin{aligned}
& \sigma_{11}=\xi p_{A}\left(1-p_{A}\right), \quad \sigma_{12}=0, \quad \sigma_{13}=-\xi^{2} p_{A} \phi_{A}, \quad \sigma_{14}=-\xi(1-\xi) p_{A} \phi_{B} \\
& \sigma_{22}=(1-\xi) p_{B}\left(1-p_{B}\right), \quad \sigma_{23}=-\xi(1-\xi) p_{B} \phi_{A}, \quad \sigma_{24}=-(1-\xi)^{2} p_{B} \phi_{B} \\
& \sigma_{33}=\xi \phi_{A}\left(1-\phi_{A}\right), \quad \sigma_{34}=0, \quad \sigma_{44}=(1-\xi) \phi_{B}\left(1-\phi_{B}\right) .
\end{aligned}
$$

Proof: See Appendix C.

\section{Some Related Tests}

As a natural follow up of the proposed rule is to consider some testing problems. For these, we first set our null hypothesis as

$$
H_{01} \text { : Treatments } A \text { and } B \text { are equivalent }
$$

and the alternative hypothesis as

$$
H_{11} \text { : Treatment } A \text { is better than } B \text {. }
$$

Unlike a single period two treatment design, we here set the equivalence of $A$ and $B$ by

$$
p_{A}=p_{B} \quad \text { and } \quad \phi_{A}=\phi_{B}
$$

and that $A$ is better than $B$ by

$$
p_{A}>p_{B}, \quad \phi_{A}>\phi_{B} .
$$

Then a test for $H_{01}$ against $H_{11}$ can be carried out by using the vector

$$
\hat{\boldsymbol{\Delta}}_{n}=\left(\hat{p}_{A}-\hat{p}_{B}, \hat{\phi}_{A}-\hat{\phi}_{B}\right)^{\prime}
$$

which, under $H_{01}$ can be expressed as

$$
\left(\begin{array}{cccc}
\frac{1}{N_{1 A}(n)} & \frac{-1}{N_{1 B}(n)} & 0 & 0 \\
0 & 0 & \frac{1}{N_{2 A}(n)} & \frac{-1}{N_{2 B}(n)}
\end{array}\right) \mathbf{T}=\frac{1}{n} \hat{\mathbf{C}} \mathbf{T},
$$

say. Hence, by using Result $3.3, \sqrt{n} \hat{\boldsymbol{\Delta}}_{n}$ converges to $N_{2}\left(\mathbf{0}, \mathbf{C} \boldsymbol{\Sigma} \mathbf{C}^{\prime}\right)$ in distribution as $n \rightarrow \infty$, where

$$
\mathbf{C}=\left(\begin{array}{cccc}
\frac{1}{\xi} & \frac{-1}{1-\xi} & 0 & 0 \\
0 & 0 & \frac{1}{\xi} & \frac{-1}{1-\xi}
\end{array}\right)
$$

under $H_{01}, \xi=1 / 2$. In general $\xi$ can be estimated by replacing the unknown parameters $p_{A}, p_{B}, \phi_{A}$, and $\phi_{B}$ by their respective ML estimates. Here $\Sigma$ involves the unknown parameters $p$ and $\phi$, which are, respectively, the common values of $\left(p_{A}, p_{B}\right)$ and $\left(\phi_{A}, \phi_{B}\right)$ under $H_{01}$. Estimates (here the ML estimates) of $p$ and $\phi$ are, respectively, given by

$$
\hat{p}=\frac{1}{n} \sum_{i=1}^{n} Z_{i}, \quad \hat{\phi}=\frac{1}{n} \sum_{i=1}^{n} U_{i} .
$$


Then the statistic defined by

$$
Q_{1}=n \hat{\Delta}_{n}^{\prime}\left(\hat{\mathbf{C}} \hat{\Sigma} \hat{\mathbf{C}}^{\prime}\right)^{-1} \hat{\boldsymbol{\Delta}}_{n}
$$

has asymptotically $\chi^{2}$-distribution with 2 d.f. under $H_{01}$, where $\hat{\Sigma}$ is the estimate of $\Sigma$ with $p$ and $\phi$ replaced by $\hat{p}$ and $\hat{\phi}$, respectively. This leads us to suggest the following test. Reject $H_{01}$ asymptotically at the level $\alpha$ iff

$$
Q_{1}>\chi_{2, \alpha}^{2}
$$

where $\chi_{2, \alpha}^{2}$ is the upper $100 \alpha \%$ point of a $\chi^{2}$ distribution with 2 d.f.

To examine the empirical size (ES) for the above test of size $\alpha$, we conduct a simulation study based on 10000 simulations for various values of $p$ and $\phi$, taking sample sizes 30 and 40 and a nominal $\alpha=0.05$. Results of the simulation study are shown in Table 1 . The simulation study shows that the empirical size of the test is satisfactorily close to the nominal one, and it tends to be conservative.

Table 1: Empirical size (ES) of the test for the treatment effect

\begin{tabular}{cccc}
$p$ & $\phi$ & ES for $n=30$ & ES for $n=40$ \\
\hline 0.1 & 0.9 & 0.0474 & 0.0483 \\
0.2 & 0.8 & 0.0415 & 0.0457 \\
0.3 & 0.7 & 0.0434 & 0.0454 \\
0.4 & 0.6 & 0.0397 & 0.0435 \\
0.5 & 0.5 & 0.0418 & 0.0441 \\
0.6 & 0.4 & 0.0373 & 0.0409 \\
0.7 & 0.3 & 0.0428 & 0.0453 \\
0.8 & 0.2 & 0.0448 & 0.0456 \\
0.9 & 0.1 & 0.0457 & 0.0497 \\
\hline
\end{tabular}

To calculate the asymptotic local power of this test procedure we consider the sequence of local alternatives defined by

$$
\begin{array}{ll}
p_{A}=p+\frac{b_{1}}{\sqrt{n}}, & p_{B}=p, \quad b_{1} \geq 0 \\
\phi_{A}=\phi+\frac{b_{2}}{\sqrt{n}}, & \phi_{B}=\phi,
\end{array}
$$

with $\left(b_{1}, b_{2}\right)^{\prime} \neq \mathbf{0}$. Then, writing

$$
\boldsymbol{\mu}_{n}=\left(p_{A}-p_{B}, \phi_{A}-\phi_{B}\right)^{\prime},
$$

we get as $n \rightarrow \infty, \sqrt{n} \boldsymbol{\mu}_{n} \rightarrow\left(b_{1}, b_{2}\right)^{\prime}=\mathbf{h}_{1}$, say. Hence, we get

$$
\sqrt{n} \hat{\boldsymbol{\Delta}}_{n} \stackrel{\mathcal{D}}{\longrightarrow} N_{2}\left(\mathbf{h}_{1}, \mathbf{C} \boldsymbol{\Sigma} \mathbf{C}^{\prime}\right)
$$


as $n \rightarrow \infty$, which implies that $Q_{1}$ converges in distribution to a non-central chi-square distribution with 2 d.f. and with the non-centrally parameter $\delta_{1}$ given by

$$
\delta_{1}=\mathbf{h}_{1}^{\prime}\left(\mathbf{C} \Sigma \mathbf{C}^{\prime}\right)^{-1} \mathbf{h}_{1} .
$$

Writing $\chi_{2, \delta_{1}}^{2}$ as the corresponding non-central random variable, the local asymptotic power of the test procedure is given by

$$
P_{1}=P\left(\chi_{2, \delta_{1}}^{2}>\chi_{2, \alpha}^{2}\right) .
$$

Table 2 shows the asymptotic power (AP) of the above test only at the level 0.05 corresponding to different values of local alternatives. We have obtained, not shown here, the Q-Q plots for the distributions of the test statistic under the sequence of local alternatives taking some selected parametric points. It is seen that the distributions are close to $\chi_{2, \delta_{1}}^{2}$ when $n \geq 30$. So the limiting power can be achieved by taking a sample of size 30 or more. We see that the AP is invariant with respect to $p$ and $\phi$.

Table 2: AP of the test for treatment effect. Figures within the cells give the values of AP corresponding to $\left(b_{1}, b_{2}\right)=(1,1)$ or $(1.5,1.5)$ or $(2,2)$, respectively.

\begin{tabular}{cccc} 
& \multicolumn{3}{c}{$\phi$} \\
\cline { 2 - 4 }$p$ & 0.3 & 0.5 & 0.8 \\
\hline \multirow{3}{*}{0.3} & 0.2621 & 0.2438 & 0.2978 \\
& 0.5334 & 0.4976 & 0.5986 \\
& 0.7951 & 0.7588 & 0.8530 \\
& 0.2438 & 0.2255 & 0.2795 \\
0.5 & 0.4976 & 0.4604 & 0.5659 \\
& 0.7588 & 0.7175 & 0.8254 \\
& 0.2978 & 0.2795 & 0.3335 \\
0.8 & 0.5986 & 0.5659 & 0.6575 \\
& 0.8530 & 0.8254 & 0.8962 \\
\hline
\end{tabular}

Next we consider a test for carry-over effect by setting the hypothesis $H_{02}: p_{A}=\phi_{A}$ and $p_{B}=\phi_{B}$ against the general alternative. As in the case of $H_{01}$, this test can performed by using the vector

$$
\hat{\Upsilon}_{n}=\left(\hat{p}_{A}-\hat{\phi}_{A}, \hat{p}_{B}-\hat{\phi}_{B}\right)^{\prime}
$$

Such a vector can be expressed as

$$
\left(\begin{array}{cccc}
\frac{1}{N_{1 A}} & 0 & \frac{-1}{N_{2 A}} & 0 \\
0 & \frac{1}{N_{1 B}} & 0 & \frac{-1}{N_{2 B}}
\end{array}\right) \mathbf{T}
$$

Hence, by using Result 3.3, $\sqrt{n} \hat{\Upsilon}_{n}$ converges to $N_{2}\left(\mathbf{0}, \mathbf{D} \boldsymbol{\Sigma} \mathbf{D}^{\prime}\right)$ in distribution as $n \rightarrow \infty$, where

$$
\mathbf{D}=\left(\begin{array}{cccc}
\frac{1}{\xi} & 0 & \frac{-1}{\xi} & 0 \\
0 & \frac{1}{1-\xi} & 0 & \frac{-1}{1-\xi}
\end{array}\right)
$$


with $\xi=\left(1-\pi_{B}\right) /\left(2-\pi_{A}-\pi_{B}\right)$, where $\pi_{A}$ and $\pi_{B}$ are, respectively, the values of $p_{A}$ and $p_{B}$ under $H_{02}$. Here both $\Sigma$ and $\mathbf{D}$ involve the unknown parameters $\pi_{A}$ and $\pi_{B}$. Estimates (here the ML estimates) of $\pi_{A}$ and $\pi_{B}$ are, respectively, given by

$$
\hat{\pi}_{A}=\frac{\sum_{i=1}^{n} \delta_{i} Z_{i}+\sum_{i=1}^{n} \tau_{i} U_{i}}{\sum_{i=1}^{n} \delta_{i}+\sum_{i=1}^{n} \tau_{i}}
$$

and

$$
\hat{\pi}_{B}=\frac{\sum_{i=1}^{n} \bar{\delta}_{i} Z_{i}+\sum_{i=1}^{n} \bar{\tau}_{i} U_{i}}{\sum_{i=1}^{n} \bar{\delta}_{i}+\sum_{i=1}^{n} \bar{\tau}_{i}} .
$$

Then the statistic defined by

$$
Q_{2}=n \hat{\boldsymbol{\Upsilon}}_{n}^{\prime}\left(\hat{\mathbf{D}} \hat{\mathbf{\Sigma}} \hat{\mathbf{D}}^{\prime}\right)^{-1} \hat{\boldsymbol{\Upsilon}}_{n}
$$

has asymptotically $\chi^{2}$-distribution with 2 d.f. under $H_{02}$, where $\hat{\mathbf{D}}$ and $\hat{\Sigma}$ are the estimates of $\mathbf{D}$ and $\Sigma$ with $\pi_{A}$ and $\pi_{B}$ replaced by $\hat{\pi}_{A}$ and $\hat{\pi}_{B}$, respectively. This leads us to suggest the following test. Reject $H_{02}$ asymptotically at the level $\alpha$ iff

$$
Q_{2}>\chi_{2, \alpha}^{2}
$$

where $\chi_{2, \alpha}^{2}$ is the upper $100 \alpha \%$ point of a $\chi^{2}$ distribution with 2 d.f.

Table 3: AP of the test for carryover effect. Figures within the cells gives the values of $\mathrm{AP}$ corresponding to $\left(c_{1}, c_{2}\right)=(1,1)$ or $(1.5,1.5)$ or $(2,2)$ respectively.

\begin{tabular}{cccc} 
& \multicolumn{3}{c}{$\pi_{B}$} \\
\cline { 2 - 4 }$\pi_{A}$ & 0.3 & 0.5 & 0.7 \\
\hline \multirow{3}{*}{0.3} & 0.1939 & 0.1578 & 0.3335 \\
& 0.3923 & 0.3095 & 0.6575 \\
& 0.6327 & 0.5131 & 0.8962 \\
0.5 & 0.1578 & 0.1327 & 0.2667 \\
& 0.3095 & 0.2495 & 0.5420 \\
& 0.5131 & 0.4154 & 0.8034 \\
& 0.3335 & 0.2667 & 0.5036 \\
0.7 & 0.6575 & 0.5420 & 0.8608 \\
& 0.8962 & 0.8034 & 0.9852 \\
\hline
\end{tabular}

To calculate the asymptotic local power of this test procedure we consider the sequence of local alternatives defined by

$$
\begin{aligned}
& p_{A}=\pi_{A}+\frac{c_{1}}{\sqrt{n}}, \quad \phi_{A}=\pi_{A}, \quad c_{1} \geq 0, \\
& p_{B}=\pi_{B}+\frac{c_{2}}{\sqrt{n}}, \quad \phi_{B}=\pi_{B}, \quad c_{2} \geq 0
\end{aligned}
$$

with $\left(c_{1}, c_{2}\right)^{\prime} \neq \mathbf{0}$. Then, writing

$$
\boldsymbol{\nu}_{n}=\left(p_{A}-\phi_{A}, p_{B}-\phi_{B}\right)^{\prime}
$$


we get, as $n \rightarrow \infty, \sqrt{n} \boldsymbol{\nu}_{n} \rightarrow\left(c_{1}, c_{2}\right)^{\prime}=\mathbf{h}_{2}$, say. Hence, we get

$$
\sqrt{n} \mathbf{\Upsilon}_{n} \stackrel{\mathcal{D}}{\longrightarrow} N_{2}\left(\mathbf{h}, \mathbf{D} \Sigma \mathbf{D}^{\prime}\right)
$$

as $n \rightarrow \infty$, which implies that $Q_{2}$ converges in distribution to a non-central chi-square distribution with 2 d.f. and with the non-centrally parameter $\delta_{2}$ given by

$$
\left.\delta_{2}=\mathbf{h}_{2} \mathbf{D} \Sigma \mathbf{D}^{\prime}\right)^{-1} \mathbf{h}_{2} .
$$

Writing $\chi_{2, \delta_{2}}^{2}$ as the corresponding non-central random variable, the local asymptotic power of the test procedure is given by

$$
P_{2}=P\left(\chi_{2, \delta_{2}}^{2}>\chi_{2, \alpha}^{2}\right) .
$$

Table 3 shows the asymptotic power (AP) of the test procedure only at the level 0.05 corresponding to different choices of local alternatives. By a similar argument as in the case of $H_{01}$, this power can be achieved by taking a sample of size 30 or more. We see that the AP is invariant with respect to $\pi_{A}$ and $\pi_{B}$.

\section{Performance Characteristics}

In this section we report the results of an extensive simulation study to find the proportions of patients receiving treatment combinations $A A, A B, B A$ and $B B$ (with the corresponding standard errors (s.e.'s).

The proportions of cases of $A A, A B, B A$, and $B B$ (with their s.e.'s in parentheses) are simulated for various choices of $\left(p_{A}, p_{B}, \phi_{A}, \phi_{B}\right)$ and $n$ with $\gamma=\beta=1$. But, in Table 4 , we report a few of these simulated results based on 10000 simulations with $n=100$ for the sake of brevity. It is observed that a considerably larger proportion of $A A$ occurs when $p_{A}>p_{B}, \phi_{A}>\phi_{B}$. This justifies the proposed design from the ethical point of view.

From Result 3.2, the limiting allocation proportions to the four treatment sequences $A A, A B, B A$, and $B B$ are as follows:

$$
\xi^{2}, \quad \xi(1-\xi), \quad \xi(1-\xi), \quad(1-\xi)^{2} .
$$

From the above limiting proportions we see that, whenever $A$ is better than $B$ (that is, under $p_{A}>p_{B}$ and $\left.\phi_{A}>\phi_{B}\right)$,

$$
\xi^{2}>(1-\xi)^{2}
$$

which indicates that the proposed design, even asymptotically, is skewed in favor of the better treatment.

Note that, when treatments $A$ and $B$ are equivalent, the limiting allocation proportions to the four sequences $A A, A B, B A$, and $B B$ are all equal to 0.25 . This in turn implies that the proposed design is asymptotically balanced which is the same as in the case of a single period RPW rule.

Another important point is that, in case of no carry-over or treatment-period interaction effect (that is, under $p_{A}=\phi_{A}$ and $p_{B}=\phi_{B}$ ), the limiting allocation proportions 
Table 4: Proportions of allocations (with s.e. in parentheses).

\begin{tabular}{cccccccc} 
& & & & \multicolumn{4}{c}{ Proportions (s.e.) of } \\
\cline { 5 - 8 }$p_{A}$ & $p_{B}$ & $\phi_{A}$ & $\phi_{B}$ & $A A$ & $A B$ & $B A$ & $B B$ \\
\hline 0.5 & 0.5 & 0.5 & 0.5 & 0.2561 & 0.2454 & 0.2462 & 0.2523 \\
& & & & $(0.0628)$ & $(0.0426)$ & $(0.0427)$ & $(0.0631)$ \\
0.5 & 0.3 & 0.5 & 0.3 & 0.3391 & 0.2412 & 0.2413 & 0.1784 \\
& & & & $(0.0587)$ & $(0.0431)$ & $(0.0428)$ & $(0.0454)$ \\
0.8 & 0.3 & 0.8 & 0.3 & 0.5830 & 0.1769 & 0.1776 & 0.0625 \\
& & & & $(0.0844)$ & $(0.0444)$ & $(0.0446)$ & $(0.0334)$ \\
0.7 & 0.4 & 0.7 & 0.4 & 0.4363 & 0.2198 & 0.2202 & 0.1237 \\
& & & & $(0.0829)$ & $(0.0442)$ & $(0.0443)$ & $(0.0486)$ \\
0.8 & 0.4 & 0.8 & 0.4 & 0.5371 & 0.1909 & 0.1921 & 0.0799 \\
& & & & $(0.0935)$ & $(0.0458)$ & $(0.0462)$ & $(0.0421)$ \\
0.3 & 0.3 & 0.3 & 0.3 & 0.2513 & 0.2492 & 0.2488 & 0.2507 \\
& & & & $(0.0461)$ & $(0.0435)$ & $(0.0433)$ & $(0.0458)$ \\
0.7 & 0.5 & 0.7 & 0.5 & 0.3837 & 0.2295 & 0.2305 & 0.1563 \\
& & & & $(0.0896)$ & $(0.0441)$ & $(0.0448)$ & $(0.0611)$ \\
0.9 & 0.5 & 0.9 & 0.5 & 0.6234 & 0.1594 & 0.1606 & 0.0566 \\
& & & & $(0.1201)$ & $(0.0526)$ & $(0.0526)$ & $(0.435)$ \\
0.7 & 0.6 & 0.7 & 0.6 & 0.3249 & 0.2377 & 0.2378 & 0.1996 \\
& & & & $(0.0949)$ & $(0.0443)$ & $(0.0442)$ & $(0.0768)$ \\
0.8 & 0.6 & 0.8 & 0.6 & 0.4212 & 0.2198 & 0.2191 & 0.1399 \\
& & & & $(0.1169)$ & $(0.0472)$ & $(0.0476)$ & $(0.0725)$ \\
0.7 & 0.7 & 0.7 & 0.7 & 0.2597 & 0.2389 & 0.2395 & 0.2619 \\
& & & & $(0.0976)$ & $(0.0438)$ & $(0.0441)$ & $(0.0982)$ \\
\hline
\end{tabular}

corresponding to each treatment will remain same for both the periods. For treatment $A$, such proportion is

$$
\xi=\frac{q_{B}}{q_{A}+q_{B}},
$$

which is analogous to the case of a single period RPW rule studied by Wei and Durham (1978). In case of no carry-over effect, although the limiting proportions of allocations corresponding to the treatment combinations $A B$ and $B A$ are same, the design is still ethical in favor of the better treatment.

In Section 4 we see that the statistic $Q_{1}$ for testing $H_{01}$ has asymptotically $\chi^{2}$-distribution with 2 d.f. under $H_{01}$. Quite naturally, the small sample distribution of $Q_{1}$ will be different from $\chi^{2}$. Here we carry out a simulation study to investigate the small sample distribution of $Q_{1}$. It is customary to investigate the null distribution of such test statistic. This is however of limited interest with adaptive designs, where we require design for skewed allocation. Accordingly we investigate the "pseudo-null" distribution by subtracting a known value of $\boldsymbol{\Delta}=\left(p_{A}-p_{B}, \phi_{A}-\phi_{B}\right)^{\prime}$ from $\hat{\boldsymbol{\Delta}}_{n}$ and consider the statistic

$$
Q_{1}^{*}=n\left(\hat{\boldsymbol{\Delta}}_{n}-\boldsymbol{\Delta}\right)^{\prime}\left(\hat{\mathbf{C}} \hat{\Sigma} \hat{\mathbf{C}}^{\prime}\right)^{-1}\left(\hat{\boldsymbol{\Delta}}_{n}-\boldsymbol{\Delta}\right) .
$$

We use a chi-square Q-Q (quantile-quantile) plot to check whether the asymptotic distribution of $T_{n}^{*}$ is chi-square with 2 degrees of freedom. The Q-Q plot plots the empirical 

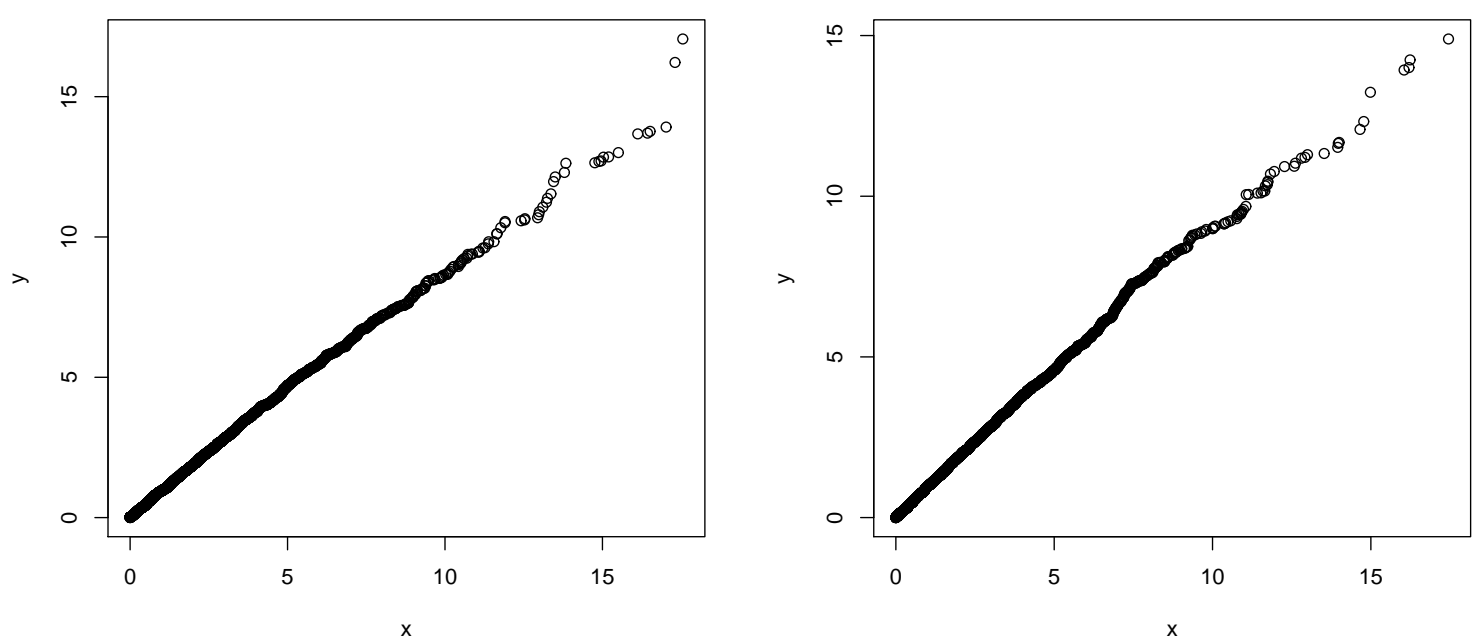

Figure 1: $\chi^{2} \mathrm{Q}-\mathrm{Q}$ plots for $n=30$ (left) and $n=40$ (right)

quantiles against the theoretical quantiles for the $\chi^{2}$-distribution. When the distribution of the variable under examination has the same shape as the reference distribution, the $\chi^{2}$ distribution in this case, the Q-Q plot is linear. In Figure 1, along the X-axis we plot the sample quantiles of the distribution of $Q_{1}^{*}$ and along the y-axis the corresponding quantiles of the $\chi^{2}$-distribution with 2 d.f. taking $p_{A}=\phi_{A}=0.6$ and $p_{B}=\phi_{B}=0.4$ with $n=30$ and 40 . From the plot, we see that there is no evidence of any strong departure from the $\chi^{2}$-distribution with 2 d.f. Hence, for small sample size a standard $\chi^{2}$ test could be used straightforwardly for inference. The adaptive nature of the design does not affect our inference.

\section{An Example}

In this section, we describe the applicability of the proposed adaptive crossover design in practice. Matthews (1989) provided some data on a three-period crossover trial of two anti-hypertensive agents. This data set was also analyzed earlier by Ebbutt (1984). The design allocated $m=17$ patients to each of the sequences $A B B, B A A, A B A, B A B$, where $A$ and $B$ denote treatment with metroprolol and metroprolol with chlorthalidone, respectively. Thus the total number of patients is $n=68$. The response is the systolic blood pressure at the end of each of the treatment periods. Our design is a two-period design with a possibility of each of $A A, A B, B A$, and $B B$. Hence we consider the data for the last two time periods of the three-period data provided by Matthews (1989). As the original data is continuous, we dichotomize it by setting a threshold of 135, i.e., a response $\leq 135$ is treated as a success, and otherwise it is a failure. From the data, we have $\hat{p}_{A}=0.2353, \hat{p}_{B}=0.2353, \hat{\phi}_{A}=0.2353$, and $\hat{\phi}_{B}=0.3529$. Treating these as the true values of $p_{A}, p_{B}, \phi_{A}$, and $\phi_{B}$, we carry out a simulation of 10000 repetitions. The simulated expectation (s.d.) of $S_{A A}$ is 15.7468 (0.0352), that of $S_{A B}$ is 16.9247 (0.0357), that of $S_{B A}$ is $17.0099(0.0359)$, and that of $S_{B B}$ is $18.3186(0.0368)$. Here 
$S_{x y}$ denotes the number of patients assigned to the treatment sequence $(x y)$. If we set the threshold as 140 , the estimates become $\hat{p}_{A}=0.3529, \hat{p}_{B}=0.5, \hat{\phi}_{A}=0.3529$ and $\hat{\phi}_{B}=$ 0.5294. Consequently,our simulation provides the expectations (s.d.'s) as $S_{A A}: 12.9984$ (0.0403), $S_{A B}$ : 16.4244 (0.0353), $S_{B A}: 16.4614$ (0.0356), $S_{B B}: 22.1158$ (0.0502). Thus, we observe that our proposed design could allocate ethically for the present data set, as the proportion of patients receiving $B B$ is much larger than the proportion of patients receiving $A A$ (here $B$ is the better treatment as it clear from the estimates of $p_{A}, p_{B}, \phi_{A}$, and $\left.\phi_{B}\right)$. Note that the proportion of patients receiving $A B$ or $B A$ has one allocation to $A$ and one allocation to $B$. Thus, in total, a considerably larger allocation could be to treatment $B$. Thus, such an ethically appropriate adaptive crossover design can easily be used in practice.

\section{Discussion}

If we look at the s.e.'s of the allocation proportions in Table 3 we observe that these s.e.'s are quite high for large values of the success probabilities. This is due to the fact that RPW rule is quite variable for large values of $p_{A}$ and $p_{B}$ and the present design is concerned with the repeated applications of the RPW rule. In the classical adaptive design the variability is reduced by application of the drop-the-loser rule, as introduced by Ivanova (2003), which at the same time has the same limiting allocations as the RPW rule. So it is expected that, for the adaptive crossover trial, the variability can be reduced by employing the drop-the-loser rule repeatedly. If we did this, we might face considerable additional difficulty to implement the rule along with various mathematical developments associated with the rule. The details are under study.

As a possible competitor of the proposed design, we may consider a non-adaptive design where each of the entering units is to receive one of the sequences $A A, A B, B A$, and $B B$, each with probability $1 / 4$. Then all the asymptotic results are also valid here with a modification that $\xi=1 / 2$. The present design has the same AP as the equal allocation design. This is due to the fact that the asymptotic distribution of $Q_{1}$ is same for both the allocations under $H_{0}$ and a sequence of local alternatives as described by (4.6)-(4.7).

The proposed procedure can easily be generalized for more than two periods but its practical usefulness is much less than two period design. So at present we do not discuss it in detail.

\section{Appendix A}

Proof of Result 3.1 : As in Melfi, Page, and Geraldes (2001), it can be seen that, almost surely, as $n \rightarrow \infty$

$$
\frac{1}{n} \sum_{k=1}^{n} \delta_{k}-\frac{1}{n} \sum_{k=1}^{n} \mathrm{E}\left(\delta_{k} \mid \delta_{(k-1)}, Z_{(k-1)}, \tau_{(k-1)}, U_{(k-1)}\right) \rightarrow 0
$$


which, using (3.1) and by martingale convergence theorem together with Kronecker's lemma, gives

$$
\begin{gathered}
\frac{N_{1 A}(n)}{n}-\frac{1}{2}\left[p_{A} \frac{1}{n} \sum_{k=1}^{n} \frac{N_{1 A}(k)}{k}+q_{B} \frac{1}{n} \sum_{k=1}^{n} \frac{N_{1 B}(k)}{k}\right. \\
\left.+\phi_{A} \frac{1}{n} \sum_{k=1}^{n} \frac{N_{2 A}(k)}{k}+\psi_{B} \frac{1}{n} \sum_{k=1}^{n} \frac{N_{2 B}(k)}{k}\right] \rightarrow 0
\end{gathered}
$$

almost surely as $n \rightarrow \infty$. Similarly, interchanging the roles of $A$ and $B$, almost surely, as $n \rightarrow \infty$,

$$
\begin{aligned}
& \frac{N_{1 B}(n)}{n}-\frac{1}{2}\left[p_{B} \frac{1}{n} \sum_{k=1}^{n} \frac{N_{1 B}(k)}{k}+q_{A} \frac{1}{n} \sum_{k=1}^{n} \frac{N_{1 A}(k)}{k}\right. \\
& \left.+\phi_{B} \frac{1}{n} \sum_{k=1}^{n} \frac{N_{2 B}(k)}{k}+\psi_{A} \frac{1}{n} \sum_{k=1}^{n} \frac{N_{2 A}(k)}{k}\right] \rightarrow 0 .
\end{aligned}
$$

As, for $i=1,2$,

$$
0 \leq \frac{N_{i A}(n)}{n} \leq 1 \quad \text { and } \quad 0 \leq \frac{N_{i B}(n)}{n} \leq 1
$$

there exist $\theta_{i}$ and $\phi_{i}$ on $[0,1]$ such that, with probability one,

$$
\lim _{n \rightarrow \infty} \sup \frac{N_{i A}(n)}{n}=\theta_{i}, \quad \text { and } \quad \lim _{n \rightarrow \infty} \inf \frac{N_{i A}(n)}{n}=\mu_{i} .
$$

From (A.2) we have after some routine steps

$$
\mu_{1} \geq \frac{1}{2}\left[p_{A} \mu_{1}+\phi_{A} \mu_{2}+q_{B}\left(1-\theta_{1}\right)+\psi_{B}\left(1-\theta_{2}\right)\right]
$$

and

$$
\theta_{1} \leq \frac{1}{2}\left[p_{A} \theta_{1}+\phi_{A} \theta_{2}+q_{B}\left(1-\mu_{1}\right)+\psi_{B}\left(1-\mu_{2}\right)\right]
$$

almost surely. Hence, combining (A.4) and (A.5), we get

$$
\begin{gathered}
\frac{1}{2}\left[p_{A} \mu_{1}+\phi_{A} \mu_{2}+q_{B}\left(1-\theta_{1}\right)+\psi_{B}\left(1-\theta_{2}\right)\right] \leq \mu_{1} \leq \theta_{1} \\
\leq \frac{1}{2}\left[p_{A} \theta_{1}+\phi_{A} \theta_{2}+q_{B}\left(1-\mu_{1}\right)+\psi_{B}\left(1-\mu_{2}\right)\right]
\end{gathered}
$$

and hence

$$
\left(\theta_{1}-\mu_{1}\right) \leq \frac{\phi_{A}+\psi_{B}}{q_{A}+p_{B}}\left(\theta_{2}-\mu_{2}\right)
$$

Similarly, (A.3) gives

$$
\left(\theta_{1}-\mu_{1}\right) \leq \frac{\phi_{B}+\psi_{A}}{q_{B}+p_{A}}\left(\theta_{2}-\mu_{2}\right) .
$$

Interchanging the roles of first period and the second period for the two treatments in (A.2) and (A.3), we get

$$
\left(\theta_{2}-\mu_{2}\right) \leq \frac{\phi_{A}+\psi_{B}}{p_{B}+q_{A}}\left(\theta_{1}-\mu_{1}\right)
$$


and

$$
\left(\theta_{2}-\mu_{2}\right) \leq \frac{\phi_{B}+\psi_{A}}{p_{A}+q_{B}}\left(\theta_{1}-\mu_{1}\right)
$$

(A.6) and (A.8) yield

$$
\left(\theta_{1}-\mu_{1}\right)\left[1-\left(\frac{\phi_{A}+\psi_{B}}{p_{B}+q_{A}}\right)^{2}\right] \leq 0 .
$$

Similarly, (A.7) and (A.9) imply

$$
\left(\theta_{1}-\mu_{1}\right)\left[1-\left(\frac{\phi_{B}+\psi_{A}}{p_{A}+q_{B}}\right)^{2}\right] \leq 0 .
$$

It can be seen that

$$
\frac{\phi_{A}+\psi_{B}}{p_{B}+q_{A}} \lessgtr 1
$$

implies

$$
\frac{\phi_{B}+\psi_{A}}{p_{A}+q_{B}} \lessgtr 1
$$

and vice versa. Then, whenever

$$
\frac{\phi_{A}+\psi_{B}}{p_{B}+q_{A}} \lessgtr 1
$$

(A.10) and (A.11) imply $\theta_{1}=\mu_{1}$, and hence $\theta_{2}=\mu_{2}$.

If $\phi_{A}+\psi_{B}=p_{B}+q_{A}$, then the treatments $A$ and $B$ must be equivalent. Because, if $A$ is better than $B, p_{A}>p_{B}$ and $\phi_{A}>\phi_{B}$ and the inequality signs are reversed when $B$ is better than $A$. So we have $p_{A}=p_{B}$ and $\phi_{A}=\phi_{B}$. Then writing, $p_{A}=p_{B}=p(=1-q)$, $\phi_{A}=\phi_{B}=\phi(=1-\psi),($ A.2) yields

$$
\mu_{1} \geq \frac{1}{2}\left[(p-q) \mu_{1}+(\phi-\psi) \mu_{2}+q+\psi\right]
$$

whenever $p>q$ and $\phi>\psi$. Similarly, for the second time period

$$
\mu_{2} \geq \frac{1}{2}\left[(p-q) \mu_{1}+(\phi-\psi) \mu_{2}+q+\psi\right] .
$$

Comparing (A.12) and (A.13), we get

$$
\mu_{1} \geq \frac{1}{2}
$$

By a similar derivation, from (A.3) and a similar equation for the second period, we get

$$
1-\theta_{1} \geq \frac{1}{2} \Longleftrightarrow \theta_{1} \leq \frac{1}{2} \text {. }
$$

Thus (A.14) and (A.15) give

$$
\theta_{1}=\mu_{1}=\frac{1}{2} \quad \text { and } \quad \theta_{2}=\mu_{2}=\frac{1}{2} .
$$


The above equality holds also for $p<q$ and $\phi<\psi$, and hence we conclude that

$$
\theta_{1}=\mu_{1} \quad \text { and } \quad \theta_{2}=\mu_{2} .
$$

Thus, using (A.2) and by a similar argument on the second period, we get

$$
\begin{aligned}
\theta_{1} & =\frac{1}{2}\left[\theta_{1} p_{A}+\left(1-\theta_{1}\right) q_{B}+\theta_{2} \phi_{A}+\left(1-\theta_{2}\right) \psi_{B}\right], \\
\theta_{2} & =\frac{1}{2}\left[\theta_{1} p_{A}+\left(1-\theta_{1}\right) q_{B}+\theta_{2} \phi_{A}+\left(1-\theta_{2}\right) \psi_{B}\right] .
\end{aligned}
$$

Combining, we conclude

$$
\theta_{1}=\theta_{2}=\frac{q_{B}+\psi_{B}}{q_{A}+\psi_{A}+q_{B}+\psi_{B}}
$$

Hence the result follows.

\section{Appendix B}

Proof of Result 3.2: Proofs of (i) - (iv) follow by a similar technique. Here, for illustration, we prove (ii) only. For this we write

$$
N_{A B}(n)=\sum_{j=1}^{n} \delta_{j}\left(1-\tau_{j}\right),
$$

and use (3.1) and (3.2) to get the expression for the conditional probability of $\left[\delta_{k+1}=\right.$ $\left.1, \tau_{k+1}=0\right]$ given $\left\{\delta_{(k)}, Z_{(k)}, \tau_{(k)}, U_{(k)}\right\}$. Then applying the same technique as in the proof of Result 3.1, we see that

$$
\begin{aligned}
& \frac{N_{A B}(n)}{n}-\frac{1}{2}\left[p_{A} \frac{1}{n} \sum_{k=1}^{n} \frac{N_{1 A}(k)}{k}+\phi_{A} \frac{1}{n} \sum_{k=1}^{n} \frac{N_{2 A}(k)}{k}+q_{B} \frac{1}{n} \sum_{k=1}^{n} \frac{N_{1 B}(k)}{k}+\psi_{B} \frac{1}{n} \sum_{k=1}^{n} \frac{N_{2 B}(k)}{k}\right] \\
& \frac{1}{2}\left[p_{B} \frac{1}{n} \sum_{k=1}^{n} \frac{N_{1 B}(k)}{k}+\phi_{B} \frac{1}{n} \sum_{k=1}^{n} \frac{N_{2 B}(k)}{k}+q_{A} \frac{1}{n} \sum_{k=1}^{n} \frac{N_{1 A}(k)}{k}+\psi_{A} \frac{1}{n} \sum_{k=1}^{n} \frac{N_{2 A}(k)}{k}\right] \rightarrow 0
\end{aligned}
$$

almost surely, as $n \rightarrow \infty$. Hence, by using Result 3.1, the required result follows.

\section{Appendix C}

Proof of Result 3.3: Here we sketch the outline of the proof. For fixed constants $C_{1}, \ldots, C_{4}$, define

$$
W_{n j}=\frac{1}{\sqrt{n}}\left[C_{1} \delta_{j}\left(Z_{j}-p_{A}\right)+C_{2} \bar{\delta}_{j}\left(Z_{j}-p_{B}\right)+C_{3} \tau_{j}\left(U_{j}-\phi_{A}\right)+C_{4} \bar{\tau}_{j}\left(U_{j}-\phi_{B}\right)\right]
$$

and hence

$$
\sum_{j=1}^{n} W_{n j}=\frac{1}{\sqrt{n}} \sum_{j=1}^{4} C_{j} T_{j}
$$


The sequence $\left\{W_{n j}, 1 \leq j \leq n, n \geq 1\right\}$ forms a martingale difference array and, by Results (3.1) and (3.2), in probability as $n \rightarrow \infty$, where

$$
\begin{aligned}
\eta^{2} & =C_{1}^{2} \xi p_{A}\left(1-p_{A}\right)+C_{2}^{2}(1-\xi)^{2} p_{B}\left(1-p_{B}\right)+C_{3}^{2} \xi \phi_{A}\left(1-\phi_{A}\right)+C_{4}^{2}(1-\xi) \phi_{B}\left(1-\phi_{B}\right) \\
& +2 C_{1} C_{3} \xi^{2} p_{A} \phi_{A}+2 C_{1} C_{4} \xi(1-\xi) p_{A} \phi_{B}+2 C_{2} C_{3} \xi(1-\xi) p_{B} \phi_{A}+2 C_{2} C_{4}(1-\xi)^{2} p_{B} \phi_{B} .
\end{aligned}
$$

Hence, using the martingale central limit theorem (See Theorem 3.2 in Hall and Heyde (1980), it follows that

$$
\sum_{j=1}^{n} W_{n j} \stackrel{\mathcal{D}}{\rightarrow} N\left(0, \eta^{2}\right) .
$$

The result then follows by using the Cramér-Wold device.

\section{Acknowledgments}

The authors are grateful to the editor and the anonymous referee for their suggestions and comments. The authors also acknowledge some fruitful discussion with Prof. K. C. Carriere and her interest during the preparation of the manuscript.

\section{References}

Bandyopadhyay, U., and Biswas, A. (1996). Delayed response in randomized play-thewinner rule: a decision theoretic outlook. Calcutta Statistical Association Bulletin, 46, 69-88.

Bandyopadhyay, U., and Biswas, A. (1997). Some sequential tests in clinical trials based on randomized play-the-winner rule. Calcutta Statistical Association Bulletin, 47, 67-89.

Bandyopadhyay, U., and Biswas, A. (1999). Allocation by randomized play-the-winner rule in the presence of prognostic factors. Sankhyā, Series B, 61, 397-412.

Bandyopadhyay, U., and Biswas, A. (2001). Adaptive designs for normal responses with prognostic factors. Biometrika, 88, 409-419.

Bandyopadhyay, U., Biswas, A., and Mukherjee, S. (2007). Adaptive two-treatment twoperiod crossover design for binary treatment responses. Statistica Neerlandica, 61, 329-344.

Bandyopadhyay, U., Biswas, A., and Mukherjee, S. (2009). Adaptive two-treatment two-period crossover design for binary treatment responses incorporating carryover effects. Statistical Methods and Applications, 18, 13-33.

Bartlett, R. H., Roloff, D. W., Cornell, R. G., Andrews, A. F., Dillon, P. W., and Zwischenberger, J. B. (1985). Extracorporeal circulation in neonatal respiratory failure: A prospective randomized trial. Pediatrics, 76, 479-487.

Biswas, A., and Dewanji, A. (2004). A randomized longitudinal play-the-winner design for repeated binary data. Australian and New Zealand Journal of Statistics, 46, 675-684.

Brown, B. W. (1980). The crossover experiment for clinical trial. Biometrics, 36, 69-79.

Carriere, K. C., and Huang, R. (2000). Crossover designs for two-treatment clinical trials. Journal of Statistical Planning and Inference, 87, 125-134. 
Cox, D. R., and Snell, E. J. (1989). Analysis of Binary Data. London: Chapman and Hall.

Ebbutt, A. F. (1984). Three-period crossover designs for two treatments. Biometrics, 40, 219-224.

Geraldes, M., Melfi, V. F., Page, C., and Zhang, H. (2006). The doubly adaptive weighted diferences design. Journal of Statistical Planning and Inference, 136, 1923-1939.

Grizzle, J. E. (1965). The two-period change-over design and its use in clinical trials. Biometrics, 21, 467-480.

Hall, P., and Heyde, C. C. (1980). Martingale Limit Theory and Applications. New York: Academic Press.

$\mathrm{Hu}, \mathrm{F}$., and Rosenberger, W. F. (2006). The Theory of Response-Adaptive Randomization in Clinical Trials. New York: John Wiley and Sons.

Ivanova, A. (2003). A play-the-winner-type urn design with reduced variability. Metrika, $58,1-13$.

Ivanova, A., Rosenberger, W. F., Durham, S. D., and Flournoy, N. (2000). A birth and death urn for randomized clinical trials: asymptotic methods. Sankhy $\bar{a}$, Series B, 62, 104-118.

Jones, B., and Kenward, M. G. (2003). Design and Analysis of Crossover Trials. Boca Raton: Chapman and Hall/CRC.

Kunert, J. (1991). Crossover designs for two treatments and correlated errors. Biometrika, 78, 315-324.

Kushner, H. B. (1997). Optimality and efficiency of two-treatment repeated measurements design. Biometrika, 84, 455-468.

Liang, Y., and Carriere, K. C. (2008). The stratified and randomized play-the-winner rule. Statistical Methods in Medical Research, 17, 581-593.

Liang, Y., and Carriere, K. C. (2009). Multiple-objective response adaptive repeated measurement designs for clinical trials. Journal of Statistical Planning and Inference, 139, 1134-1145.

Matthews, J. N. S. (1989). Estimating dispersion parameters in the analysis of data from crossover trials. Biometrika, 76, 239-244.

Melfi, V. F., Page, C., and Geraldes, M. (2001). An adaptive randomized design with application to estimation. The Canadian Journal of Statistics, 29, 107-116.

Reiertsen, O., Larsen, S., and Solhaug, J. H. (1998). Development of play-the-winner design: weighting and accumulation of information. Scandinavian Journal of Clinical and Laboratory Investigation, 58, 241-250.

Reiertsen, O., Larsen, S., Størkson, R., Trondsen, E., Løvig, T., Andersen, O. K., et al. (1993). Safety of enoxaparin and dextran-70 in the prevention of venous thromboemlism in digestive surgery. a play-the-winner-designed study. Scandinavian Journal of Gastroenterology, 28, 1015-1020.

Reiertsen, O., Mowinckel, P., Bjerkeseth, O., Løvig, T., Thorsen, G., Gerner, T., et al. (1996). Characterization of 'winners' to enoxaparin in the prevention of postoperative venous thromboemlism in digestive surgery. A play-the-winner-designed study. Scandinavian Journal of Gastroenterology, 31, 616-621.

Rosenberger, W. F. (1993). Asymptotic inference with response-adaptive treatment allocation designs. Annals of Statistics, 21, 2098-2107. 
Rosenberger, W. F. (1996). New directions in adaptive designs. Statistical Science, 11, 137-149.

Senn, S. (1993). Cross-over Trials in Clinical Research. New York: John Wiley and Sons.

Taka, M. T., and Armitage, P. (1983). Autoregressive models in clinical trials. Communications in Statistics: Theory and Methods, 12, 865-867.

Tamura, R. N., Faries, D. E., Andersen, J. S., and Heiligenstein, J. H. (1994). A case study of an adaptive clinical trials in the treatment of out-patients with depressive disorder. Journal of the American Statistical Association, 89, 768-776.

Vonesh, E. F., and Chinchilli, V. M. (1996). Linear and Nonlinear Models for the Analysis of Repeated Measurements. New York: Marcel Dekker/CRC.

Wei, L. J. (1979). The generalized Polya's urn for sequential medical trials. Annals of Statistics, 7, 291-296.

Wei, L. J. (1988). Exact two-sample permutation tests based on the randomized play-thewinner rule. Biometrika, 75, 603-606.

Wei, L. J., and Durham, S. (1978). The randomized play-the-winner rule in medical trials. Journal of the American Statistical Association, 73, 838-843.

Wei, L. J., Smythe, R. T., Lin, D. Y., and Park, T. S. (1990). Statistical inference with data-dependent treatment allocation rule. Journal of the American Statistical Association, 85, 156-162.

Zelen, M. (1969). Play-the-winner rule and the controlled clinical trial. Journal of the American Statistical Association, 64, 131-146.

Authors' addresses:

Uttam Bandyopadhyay

Department of Statistics

University of Calcutta

35 Ballygunge Circular Road

Kolkata - 700019

India

ubstat@caluniv.ac.in

Atanu Biswas

Applied Statistics Unit

Indian Statistical Institute

203 B. T. Road

Kolkata - 700108

India

atanu@isical.ac.in

Shirsendu Mukherjee

Department of Statistics

Behala College

Kolkata - 700060

India

shirsendu_st@yahoo.co.in 